\title{
Preferencias estudiantiles de cursos libres sobre las modalidades de fitness o wellness según sexo
}

\section{Preferences of Students of Free Courses on the Modalities of Fitness or Wellness According to Sex \\ Preferência dos estudantes dos Cursos Livres as modalidades de Fitness ou Wellness segundo o sexo}

Irina Anchía-Umaña

Universidad Nacional

Heredia, Costa Rica ianchia@una.cr

(D) https://orcid.org/0000-0002-6984-8697

Ashley Elizondo-Monge

Universidad Nacional

Heredia, Costa Rica

ashelizondo13@gmail.com

iD https://orcid.org/0000-0002-6285-5296

Steve Monge-Poltronieri

Universidad Nacional

Heredia, Costa Rica

spmonge@hotmail.com

https://orcid.org/0000-0002-2206-2259

Inés Revuelta-Sánchez

Universidad Nacional

Heredia, Costa Rica

ines.revuelta.sanchez@una.cr

(iD) https://orcid.org/0000-0003-4560-1953

Braulio Sánchez-Ureña

Universidad Nacional

Heredia, Costa Rica

braulio.sanchez.urena@una.cr

iD https://orcid.org/0000-0001-8791-6836

Juan Carlos Gutiérrez-Vargas

Universidad Nacional

Heredia, Costa Rica jucagu@msn.com

https://orcid.org/0000-0002-0689-6771

Recibido • Received • Recebido: 24 / 11 / 2017

Corregido • Revised • Revisado: 04 / 02 / 2019

Aceptado • Accepted • Aprovado: 21 / 06 / 2019 
doi: http://dx.doi.org/10.15359/ree.23-3.10

URL: http://www.una.ac.cr/educare

CORREO: educare@una.cr

Resumen: El objetivo del presente estudio fue determinar las preferencias de matrícula según sexo, del estudiantado de cursos libres de la Escuela Ciencias del Movimiento Humano y Calidad de Vida (CIEMHCAVI) de la Universidad Nacional en Costa Rica, en cuanto a las modalidades de fitness o wellness. Procedimiento: Esta investigación se desarrolló con un enfoque retrospectivo, con registros desde el año 2012 al 2016. Participantes: se trabajó con una muestra de 2112 estudiantes con edades desde los 17 a 62 años y un $28.3 \pm 7.8$ años, (1181 hombres y 931 mujeres), estudiantado usuario de los cursos libres impartidos entre los años 2012 a 2016 en la escuela CIEMHCAVI. Las variables estudiadas fueron género, edad, curso en el que participó y nivel aprobación o reprobación. Resultados: Se encontró que existen diferencias estadísticamente significativas ( $x 2=141.916, p=$ $<.001)$ en las preferencias de la modalidad de curso según sexo, así como no existen diferencias entre cuartilos de edades $\left(\mathrm{X}_{(3)}^{2}=2,939 ., \mathrm{p}=<.40\right)$ o clasificación de género en los porcentajes de aprobación o reprobación de los cursos ( $2=.064, \mathrm{p}=<.800)$.

Palabras claves: Ciencias de la salud; Costa Rica; desarrollo social; educación continua; educación superior; género.

Abstract: The object of the present study was to determine, according to sex, the preference in courses registered by students, regarding the modalities of Fitness or Wellness in the Free-courses Programs of the Escuela Ciencias del Movimiento Humano y Calidad de Vida (CIEMHCAVI) of the Universidad Nacional in Costa Rica. Procedure: The research was conducted under a retrospective approach, with data obtained from records from the years 2012 to 2016. Participants: a sample of 2112 students aged 17 to 62 years and $28.3 \pm 7.8$ years, (1181 men and 931 women), enrolled in one or more of the Freecourses Programs taught between 2012 and 2016 at CIEMHCAVI/UNA. The variables studied were gender, age, course/s registered, and approval or disapproval. Results: There are statistically significant differences $(x 2=141.916, p=<.001)$ in the registration preferences of the modality (Fitness or Wellness) according to sex, but no differences found between quartiles of ages $(X 2(3)=2,939 ., p=<.40)$ or gender classification in the percentages of approval or failure of the courses ( $X 2=.064, p=<.800$ ).

Keywords: Health sciences; Costa Rica; social development; continued education; higher education; gender.

Resumo: O objetivo do presente estudo foi determinar as preferências de matrícula segundo o sexo dos estudantes dos cursos livres da Escola de Ciências do Movimento Humano e Qualidade de Vida (CIEMHCAVI) da Universidade Nacional na Costa Rica, quanto às modalidades de Fitness ou Wellness. Procedimento: Esta pesquisa desenvolveu-se sob um foco retrospectivo, em que foram tomados os registros desde o ano de 2012 até 2016. Participantes: Trabalhou-se com uma amostra de 2112 estudantes, com idades que variam dos 17 aos 62 anos e um $28.3 \pm 7.8$ anos, (1181 homens e 931 mulheres) usuários dos cursos livres dados entre os anos de 2012 a 2016 na escola CIEMHCAVI. As variáveis estudadas foram: gênero, idade, curso no qual participou e nível de aprovação ou reprovação. Resultados: Foi encontrado que existem diferenças estatisticamente significativas ( $x 2$ $=141.916, p=<.001)$ nas preferências da modalidade do curso segundo o sexo, assim como não existem diferenças entre subgrupos de idades $(X 2(3)=2,939 ., p=<.40)$ ou classificação do gênero nas porcentagens de aprovação ou reprovação dos cursos ( $2=.064, \mathrm{p}=<.800)$.

Palavras chave: Ciências da saúde, Costa Rica, desenvolvimento social, educação contínua, educação superior, gênero. 


\section{Introducción}

Durante el siglo XVIII existió un debate sumamente significativo en relación con las posibilidades de que las mujeres pudieran tener acceso al estudio universitario, pues estas opciones eran consideradas como pertenecientes al "territorio masculino". Esta concepción logró superarse gracias al fuerte desarrollo y movimientos de mujeres con deseos de integrarse como profesionales al campo laboral (Offen, 2000).

Es así como en la América Latina de finales del siglo XVIII e inicios del XIX, las naciones"recién nacidas" disputaban por lograr la hegemonía regional con gran dificultad para adaptarse a sus propias condiciones políticas, lo cual ocasionó guerras territoriales que estallaron por doquier y, producto de esto, hubiera nuevos intereses en la región: la patria, los grupos indígenas y la educación de la mujer (Ballesteros, 1997).

Lo anterior consistió en una verdadera "revolución", ya que tradicionalmente el derecho al estudio había estado reservado para los hombres; a las mujeres se les asignaban las labores domésticas, así como la reproducción y los cuidados de la familia (Godoy, Díaz y Mauro, 2009). Esta división del trabajo en el ámbito social, por ejemplo en cuanto a responsabilidades y actividades, son diferenciadas dependiendo si van dirigidas a hombres o mujeres, ya sea a nivel público o privado (Brunet y Alarcón, 2005; Cárdenas, Correa y Prado, 2013), es reproducida en el mercado laboral tanto en las profesiones, oficios y ocupaciones como también en la distribución de la fuerza de trabajo en los diferentes sectores de la economía y al interior de las jerarquías organizacionales (Anker, 1997; Anker, Melkas y Korten, 2003).

El caso de Chile constituye un ejemplo puntual. A nivel universitario y desde 1877, la Universidad de Chile fue la primera institución universitaria latinoamericana que abrió sus puertas a las mujeres; esto, a veinticuatro años de haber sido fundada con una población estudiantil integrada únicamente por hombres y casi tres siglos después de la fundación de La Real y Pontificia Universidad de México (Ballesteros, 1997).

Los procesos históricos de exclusión tanto en lo que se relacionan con el acceso, así como con la formación académica, se han erradicado cada vez más gracias a la inclusión de las mujeres en este sector de estudios superiores universitarios (Ballesteros, 1997).

Elaccesoa la educación formal ha significado un aspecto degran relevancia en la consolidación de la igualdad entre hombres y mujeres (Papadópulos y Radakovich, 2006). Este espacio -que lenta, pero sostenidamente, se ha desarrollado en particular en las últimas décadas- ha implicado el aporte de conocimiento e información que retroalimenta estos esfuerzos de mayor apertura en la educación, a todos los niveles, para las mujeres. En este sentido y en particular en la actualidad, los estudios realizados en género han permitido disminuir sesgos y estigmas, así como refutar conceptos y teorías (European Commission Research \& Innovation, 2011), lo cual, indudablemente, ha contribuido con los procesos de democratización de la educación universitaria. 
doi: http://dx.doi.org/10.15359/ree.23-3.10

URL: http://www.una.ac.cr/educare

CORREO: educare@una.cr

A pesar del camino recorrido, aún falta mucho por hacer. En palabras de Antonio Manuel de Oliveira Gutiérrez, actual secretario general de las Naciones Unidas en su mensaje del Día Internacional de la Mujer (Naciones Unidas Secretario General, 2017):

Los desequilibrios históricos en las relaciones de poder entre el hombre y la mujer, agravados por el aumento de las desigualdades dentro de la sociedad y los países, ... están dando lugar a una mayor discriminación contra las mujeres y las niñas. En todo el mundo, la tradición, los valores culturales y la religión se utilizan indebidamente para restringir los derechos de las mujeres, afianzar el sexismo y defender las prácticas misóginas. (párr., 2)

Dichas declaraciones, emitidas en pleno 2017, indican, sin lugar a dudas, que el camino recorrido no está cerca de la meta, si esta se considera como el logro de un acceso en igualdad de condiciones para las mujeres en todos los ámbitos. La declaración se sigue aplicando en el campo de la educación, pues los avances que se han producido no significan un logro definitivo sino un avance en un proceso que no solo debe continuar, sino que se debe monitorear.

En este sentido, los beneficios de la educación y particularmente a nivel de género, se deben relacionar no solo con la participación laboral, sino también con resultados que redundan en aspectos "monetarios, así como no-monetarios" del bienestar tanto individual como colectivo. En lo que respecta a los beneficios monetarios, se refiere el ingreso o retribución en salario o especia que devenga una persona por su trabajo y que influye en su estatus económico y social. Por otra parte, el beneficio "no-monetario" para las personas en términos generales reside mayoritariamente en que contribuye a mejorar su bienestar físico, emocional y mental. Estos beneficios "no- monetarios" en lo inmediato redundan en una mayor productividad en los ámbitos personales, de trabajo y estudio; además, los beneficios "no-monetarios" en la salud de los individuos constituyen la reducción de accidentes, la violencia y las enfermedades y, por lo cual mejoran la salud pública tanto en sus índices como en los insumos requeridos y, por ende, sus resultados a todo nivel (Organization for Economic Cooperation and Development [OECD], 2007).

Lo anterior también beneficia la economía social, pues implica menos gastos gubernamentales para velar por la salud poblacional y una mayor recaudación de los impuestos establecidos (OECD, 2007). Es así como existe una correlación entre desarrollo humano, educación y género que se debe tener presente en todo momento y formar parte del monitoreo que se debe desarrollar en relación con este tema.

En el informe más reciente del Programa de las Naciones Unidas para el Desarrollo (PNUD, 2016), Costa Rica se encuentra en el puesto 66 del ranking mundial 2015 en el listado de índices de desarrollo humano (IDH); asimismo, este se encuentra ubicado dentro de la segunda categorización más alta, denominada desarrollo humano alto. Aunado a lo anterior, en latinoamericano, Costa Rica se encuentra ubicada en el puesto número tres, después de Uruguay (puesto 54) y Panamá (puesto 60), en dicha categorización (desarrollo humano alto).

4 Irina Anchía-Umaña, Ashley Elizondo-Monge, Steve Monge-Poltronieri, Inés Revuelta-Sánchez, Braulio Sánchez-Ureña y Juan Carlos Gutiérrez-Vargas

Los artículos de la Revista Electrónica Educare del Centro de Investigación y Docencia en Educación de la Universidad Nacional, Costa Rica, se comparten bajo términos de la Licencia Creative Commons: Reconocimiento, No Comercial, Sin Obra Derivada 3.0 Costa Rica. Las autorizaciones adicionales a las aquí delimitadas se pueden obtener en el correo: educare@una.cr 
En relación con la educación superior en América Latina, en el periódico El País, en el año 2014, se expuso, por primera vez en la historia, que más de la mitad de las mujeres (representando el 52,9\%) se ubican dentro del campo laboral en América Latina; lo anterior es producto de un aumento en las oportunidades de estudio con las que se cuenta en el momento.

En forma adicional, el 53,7 \% de las mujeres latinoamericanas tienen diez o más años de estudiar o ejercer, contrastan así los indicadores definidos para los hombres, los cuales presentan índices del $40 \%$ y $16 \%$. Estos datos fueron publicados por la Oficina Regional del Trabajo para América Latina y el Caribe (Varela, 2014).

El informe de la Organización de las Naciones Unidas para la Educación, la Ciencia y la Cultura (UNESCO, 2015) sobre la ciencia, expone que las tres regiones con una cantidad mayor de mujeres investigadoras son Europa Sudoriental con un 49 \%, así como el Caribe, Asia Central y América Latina con un $44 \%$, por lo que Costa Rica se encuentra dentro de este marcador (Soto, 2017.

Al respecto y relacionando lo anterior con la existencia de instituciones educativas universitarias que ofrezcan el espacio para desarrollarse no solo en el ámbito de la ciencias, sino del conocimiento en general, cabe señalar que en Costa Rica, en la primera parte de la década del 2010, se reportan 63 instituciones universitarias, las cuales se conforman de la siguiente manera: cinco estatales públicas, cinco internacionales y 53 privadas. Además de lo anterior, se cuenta con instituciones para-universitarias de las cuales dos son estatales y 27 son privadas (Consejo Nacional de Rectores [CONARE], 2013); lo anterior suma 92 instituciones en total, las cuales ofrecen una oferta académica realmente amplia. Los datos no han cambiado significativamente y a la fecha Costa Rica cuenta con un sistema mixto universitario compuesto por 64 universidades, de las cuales 5 son estatales públicas, 54 privadas y 5 internacionales que ofrecen más de 1.300 programas en diversas áreas del conocimiento (Programa Estado de la Nación, 2017).

En el caso particular de las áreas de formación en wellness y fitness, se entiende la primera como todo lo que comprende la salud y un compendio en el que la actividad física, la buena alimentación, las relaciones sociales, la espiritualidad fortalecida y una relación saludable con el medio ambiente se encuentran integradas para contribuir a una verdadera y excelente calidad de vida y bienestar. Para alcanzar lo anterior, la educación y la sensibilización son bases para una vida wellness (Colado, 2000). Con respecto al fitness, este se conceptualiza como la consecución y la estabilización de la salud mediante el ejercicio físico, para sentirse bien gracias al buen ver del cuerpo (Corrales, 2010). Si bien es cierto que ambas áreas pertenecen al ámbito de la salud y de las ciencias del movimiento humano, los principios que guían a practicantes de cada una de ellas se basan en orientaciones y objetivos diferentes.

Es importante, para efecto de ubicación del espacio académico universitario en el cual se ha elaborado este estudio, indicar que la Universidad Nacional (UNA) es una institución estatal pública de enseñanza universitaria, que cuenta con varias facultades y entre ellas, la Facultad de Ciencias de la Salud en la cual se ubica la Escuela de Ciencias del Movimiento Humano y 
doi: http://dx.doi.org/10.15359/ree.23-3.10

URL: http://www.una.ac.cr/educare

CORREO: educare@una.cr

Calidad de Vida (CIEMHCAVI), unidad académica que oferta carreras en ciencias del movimiento humano y calidad de vida.

Entre sus postulados, la CIEMHCAVI establece como objetivo formar profesionales con la capacidad de responder a las necesidades de las personas en el área de la salud, la educación y el movimiento humano; esto, con bases científicas y habilidades emocionales para contribuir con la promoción de la salud por medio de la actividad física mediante un trabajo interdisciplinario con un fuerte componente práctico-biopsicosocial. Ello, con el fin de que estos grupos profesionales preserven y promuevan la salud previniendo la enfermedad desde lo emocional hasta lo físico.

Como parte del concepto integrador en el campo de la academia, la UNA y la CIEMHCAVI desarrollan programas y proyectos cuyos fines se establecen relacionados con las áreas de docencia, extensión, investigación y producción que guían su accionar el cual se dirige hacia la sociedad. En el área específica de la extensión relacionada con la proyección hacia la comunidad, la UNA ha establecido esfuerzos en diferentes niveles, sobre todo en las ciencias y el arte, la realización y formulación de proyectos, de germinación de empresas y de cursos de capacitación (Programa Estado de la Nación, 2015).

Como parte de los programas de extensión y sus correspondientes cursos de capacitación que se ejecutan en la CIEMHCAVI, se encuentran el Centro de Desarrollo y Rehabilitación de la Salud (CEDERSA) y el Programa de Ciencias del Ejercicio y la Salud (PROCESA). A partir de estos se realiza la investigación base de este artículo. En estos programas, y como parte de las funciones desarrolladas particularmente en el ámbito de la extensión universitaria y su proyección hacia la comunidad en general, se ofrecen cursos libres entre los cuales y para el caso de PROCESA y CEDERSA, en su oferta se cuenta con los de wellness así como fitness.

Atendiendo las ideas y datos formulados anteriormente, se ha planteado como problema de estudio las desigualdades que se presentan entre géneros con base en preferencias en el momento de matricular cursos y acceder a la oferta académica, lo cual podría incidir en la participación de hombres y mujeres a nivel universitario.

Resulta importante estudiar y analizar, a partir de una perspectiva de construcción de género, el diseño de la oferta académica a nivel de cursos libres, con el fin de fomentar la matrícula tanto de mujeres como de hombres por igual, para propiciar los principios de igualdad y equidad al no presentar discriminación por sexo en los cursos de educación no formal.

Si bien, en el estudio se considera el ámbito de dos tipos de cursos libres, y entendiendo, por lo tanto, que su estudiantado no cursa una carrera regular, el acceso y la matrícula de estudiantes mujeres y hombres en cursos libres universitarios ofrece una capacitación que impacta, de alguna manera, en aspectos monetarios y no- monetarios, al incidir en la calidad de vida de las personas estudiantes de estos.

6 Irina Anchía-Umaña, Ashley Elizondo-Monge, Steve Monge-Poltronieri, Inés Revuelta-Sánchez, Braulio Sánchez-Ureña y Juan Carlos Gutiérrez-Vargas

Los artículos de la Revista Electrónica Educare del Centro de Investigación y Docencia en Educación de la Universidad Nacional, Costa Rica, se comparten bajo términos de la Licencia Creative Commons: Reconocimiento, No Comercial, Sin Obra Derivada 3.0 Costa Rica. Las autorizaciones adicionales a las aquí delimitadas se pueden obtener en el correo: educare@una.cr 
Es así como el problema sustantivo que preocupa atender en este estudio consiste en determinar si existe una preferencia en particular por género hacia los cursos de Fitness o de Wellness, así como el porcentaje de aprobación y reprobación en estos. Se debe tener presente que el índice de reprobación/aprobación puede impactar las preferencias del estudiantado en el momento de matricular los siguientes cursos libres o en general.

Lo anterior, por cuanto podría facilitarse a futuro y con base en los comportamientos e intereses por género, el adecuar y diseñar la oferta académica de los cursos en estas temáticas, de modo mujeres y hombres cuenten con la posibilidad tanto en cantidad como en temáticas ofrecidas, de matricular cursos que verdaderamente se adapten a sus intereses, lo cual, en forma adicional, podría coadyuvar a nivelar cada vez más el estatus "monetario y no-monetario" y reducir la brecha laboral y del conocimiento entre mujeres y hombres.

\section{Metodología}

Tipo de estudio: se trata de un estudio con un enfoque retrospectivo de tipo descriptivo y comparativo, en el que se analizan los datos registrados desde el año 2012 al 2016 de las matrículas de estudiantes de la oferta de cursos libres ofertados.

Participantes: Para el presente estudio se trabajó con una muestra de 2112 personas, con un rango de edad de 17 a 62 años y un $28.3 \pm 7.8$ años, el $55.8 \%$ (1181) son hombres y el 44,2 $\%$ (931) mujeres. Cada persona fue participante de los cursos libres que se impartieron entre los años 2012 a 2016 en los programas PROCESA Y CEDERSA de la CIEMHCAVI. En total 648 personas fueron ubicadas en la clasificación de wellness y 1464 personas a la de fitness.

Como criterio de exclusión se estableció que quienes no presentaban los datos completos (es decir, las variables tomadas en cuenta) se eliminarían de la base de datos utilizada para el análisis estadístico.

Procedimiento: Para fines de la presente investigación se agruparon los registros de la muestra según el curso en el que participaron en wellness o fitness, los datos se obtuvieron de las bases de datos de los programas CEDERSA y PROCESA. Dada la naturaleza de la fuente de información, se imposibilita hablar de criterios de validez y confiabilidad, pues la información no se obtuvo con instrumentos, sino de bases de datos descriptivos analizados de forma retrospectiva.

El grupo de fitness está compuesto por participantes de los siguientes cursos libres:

- Contrarresistencia en sus tres niveles (básico, intermedio y avanzado)

- Entrenamiento funcional en sus dos niveles (nivel básico y nivel intermedio).

- Pilates formación de instructores en sus dos niveles (nivel básico y nivel intermedio).

- Evaluación funcional del cliente en el entrenamiento personal. 
doi: http://dx.doi.org/10.15359/ree.23-3.10

URL: http://www.una.ac.cr/educare

CORREO: educare@una.cr

El grupo de wellness se encuentra conformado por participantes de los siguientes cursos libres:

- Aeróbicos formación de instructores en sus tres niveles (nivel básico, intermedio y avanzado)

- Instructor de baile popular para centros de acondicionamiento físico.

- Masaje terapéutico

- Masoterapia aplicada al deporte

- Fundamentos de terapias complementarias I

- Terapias de bienestar

- Nutrición: El camino hacia el wellness

- Nutrición aplicada a la práctica deportiva

- Yoga y meditación

- Yoga intermedio

Seguidamente se procedió a la realización de una base de datos, la cual fue alimentada con la información recopilada de los grupos participantes seleccionados. Las variables tomadas en cuenta fueron género, edad, la clasificación según el curso en el que participó cada persona (fitness o wellness) y si el sujeto participante aprobó o reprobó el curso.

\section{Análisis estadístico}

Se utilizó estadística descriptiva (promedios y desviación estándar) para la caracterización de la muestra en cuanto a su edad. Se realizaron análisis de independencia entre las variables categóricas mediante prueba de $\mathrm{X}^{2}$, contrastando el sexo en cuanto al área de interés de los cursos (fitness y wellness), así como el nivel de aprobación y reprobación de los cursos según sexo; de igual manera el nivel de aprobación y reprobación por cuartillos de edad. Los análisis se realizaron mediante el paquete estadístico para las ciencias sociales SPSS (V. 24, SPSS, Inc., Chicago, IL.), el valor de alfa para la toma de decisiones estadísticas fue de $\mathrm{p}<.05$.

\section{Resultados}

La Figura 1 evidencia la comparación según sexo por área de interés. En el análisis de datos del presente estudio se determinó que sí existen diferencias estadísticamente significativas entre la cantidad de hombres y de mujeres, según los cursos libres pertenecientes a las modalidades de fitness o wellness $\left(X_{(3)}=141.916, p=<.001\right)$. Además, según lo muestra dicha figura, tanto hombres como mujeres poseen una preferencia mayor por los cursos de la categoría Fitness sobre los cursos en la categoría Wellness, es decir, que esta tendencia se da sin distinción por sexo. 


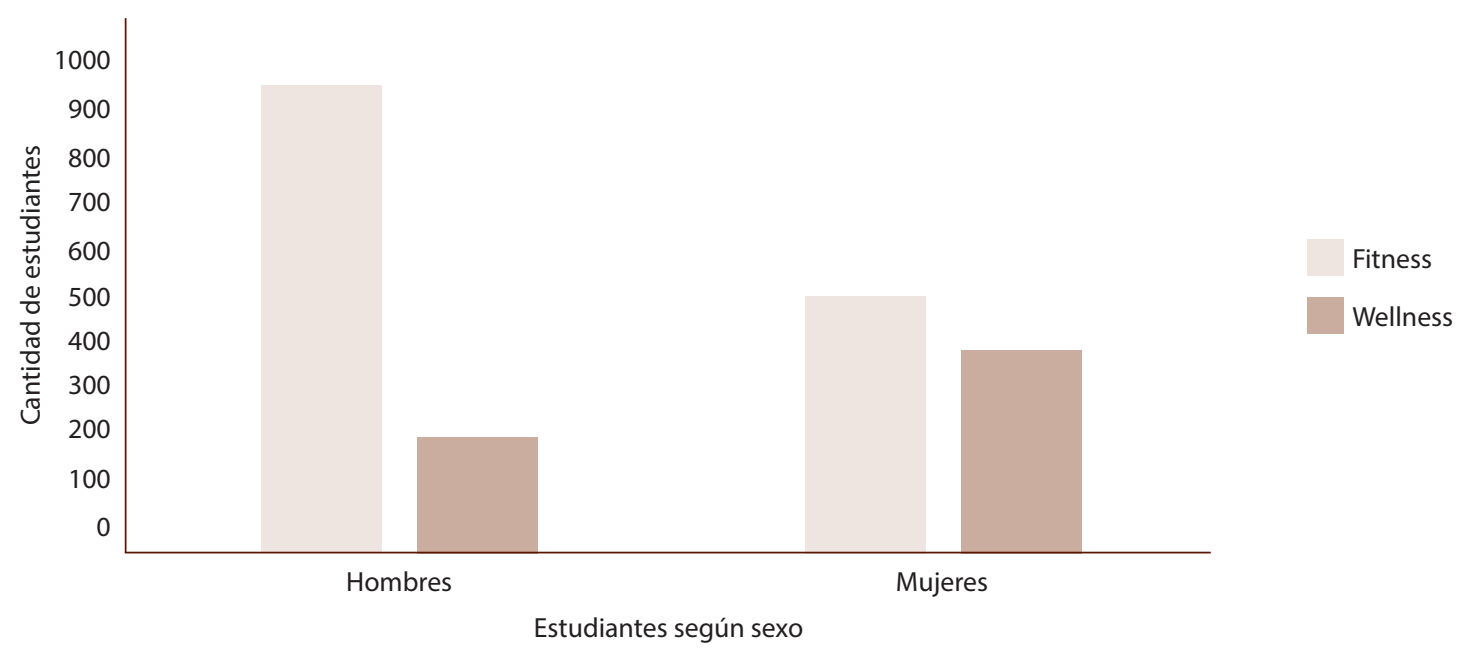

Figura 1: Distribución según género entre las clasificaciones de wellness y fitness del estudiantado de educación continua de Cursos Libres de la Universidad Nacional de Costa Rica.

Nota: Elaboración propia.

En la Figura 2, se muestra el contraste entre nivel de aprobación y reprobación según sexo. Se determinó que no existen diferencias estadísticamente significativas entre la cantidad de mujeres $\mathrm{y}$ de hombres que aprueban, o bien, reprueban los cursos libres $\left(x 2_{(3)}=.064, \mathrm{p}=\right.$ $<.800)$, lo que indica que tanto hombres como mujeres aprueban en cantidades similares los cursos a los que se inscriben, el porcentaje de aprobación en ambos grupos es mayor que el de reprobación en estos mismos en los proyectos de educación continua de los programas CEDERSA y PROCESA.

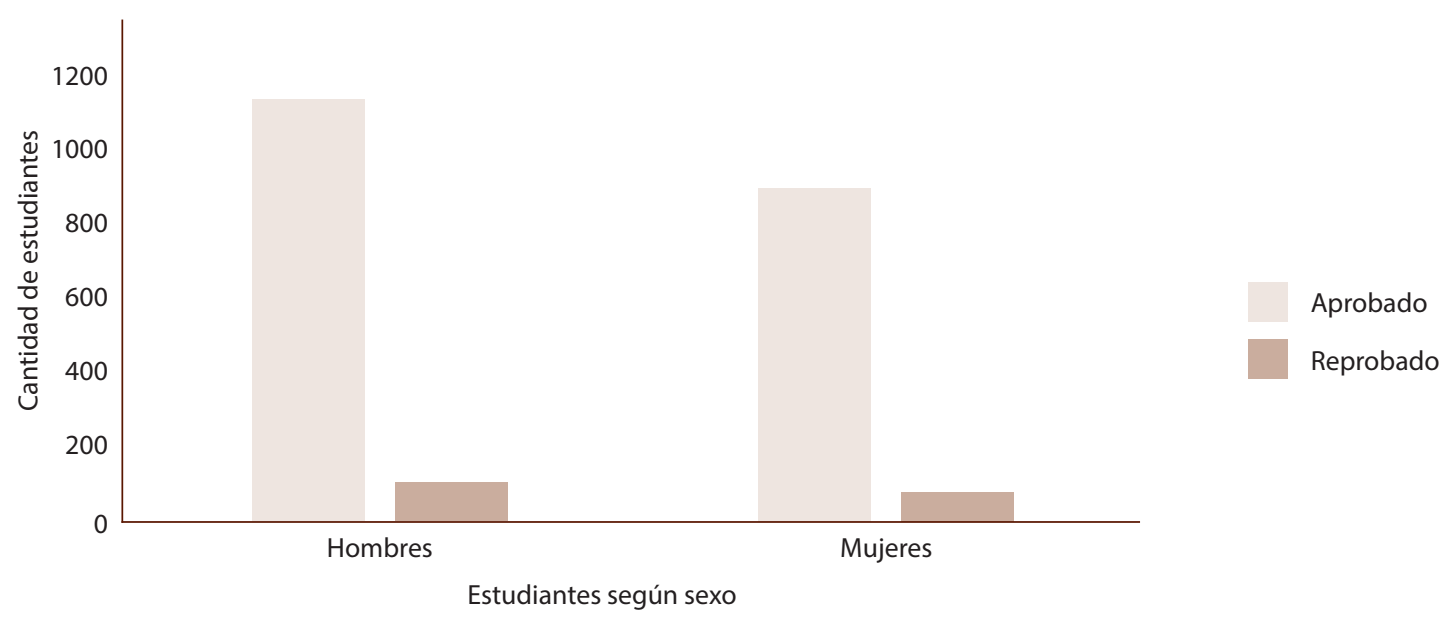

Figura 2: Nivel de aprobación y reprobación de los cursos libres de la Universidad Nacional según sexo. Nota: Elaboración propia. 
doi: http://dx.doi.org/10.15359/ree.23-3.10

URL: http://www.una.ac.cr/educare

CORREO: educare@una.cr

El análisis de los datos indica que no se encontraron diferencias estadísticamente significativas entre los cuartilos de edades en función del nivel de aprobación en el estudiantado de educación continua $\left(X_{(3)}^{2}=2,939\right.$., $\left.p=<.40\right)$. Tal como se observa en la Figura 3, indistintamente de la edad y de la clasificación, ya sea fitness o wellness, en cada agrupación se dio una mayor cantidad de estudiantes que aprobaron los cursos que quienes los reprobaron.

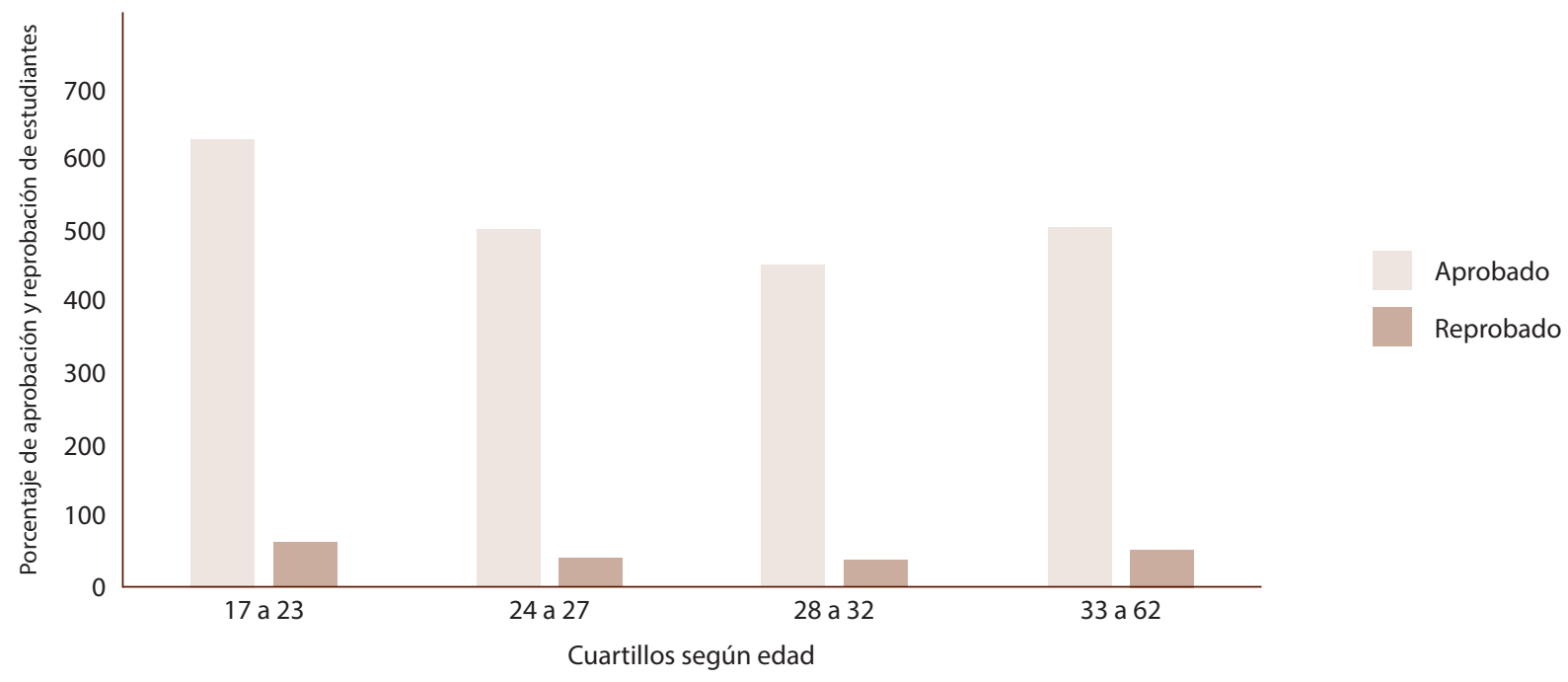

Figura 3: Porcentaje de aprobación y reprobación según los cuartilos de edades del estudiantado de educación continua de CEDERSA y PROCESA.

Nota: Elaboración propia.

\section{Discusión}

El objetivo del presente estudio fue determinar la existencia de una relación entre el sexo del estudiantado y la elección de los cursos libres de acuerdo con su categoría, ya sea wellness o fitness. Inicialmente se planteó la hipótesis de que los varones preferían sobre las mujeres los cursos de fitness y que estas, tenían una mayor inclinación por los de wellness; sin embargo, entre los hallazgos más relevantes de esta investigación, se encontró que, sin distinción de sexo, existe una mayor inclinación por los cursos enfocados en fitness, a pesar de que los asistentes de categoría wellness, en mayor medida eran mujeres.

Retomando lo anterior y analizando la teoría hallada, diversos estudios (Codina y Pestana, 2012; Moreno, Martínez y Alonso, 2006; Oviedo et al., 2013) exponen que la práctica de actividad física está estereotipada a causa de las vivencias en la infancia, ya que las mujeres prefieren actividades más pasivas o musicales como baile y yoga y que muchas de estas se realizan de forma grupal; mientras que los hombres manifiestan predilección por las actividades 
individuales y de competición como, por ejemplo, aquellas de tipo deportivo. No obstante, lo anterior no fue reflejado en los resultados de esta investigación, ya que tanto hombres como mujeres matriculan cursos en ambas modalidades en proporciones similares, tal y como se pudo observar en la Figura 1.

En contraste con lo anteriormente expuesto y los principales hallazgos de la presente investigación, por su parte, otras investigaciones (Almorza, Yébenes, Rivas y Bablé, 2010; García y Llopis, 2011; Palou, Ponseti y Borrás, 2001) han determinado una notable diferencia en cuanto a la preferencia del tipo de actividad física a realizar. Estas coinciden en que las mujeres prefieren practicar actividad física en forma individual, o bien si esta es grupal la prefieren acompañada de música, ejemplo de ello son clases de baile popular, y especialmente, a modo de estigma y como patrón repetido en ellas, prefieren las actividades con énfasis en la estética y la salud, mientras que los hombres prefieren participar de actividades que se realicen de forma grupal, o bien orientadas a la competición, preferiblemente los deportes tradicionales como el fútbol, los cuales tienen como fin la recreación y fomentar espacios para la socialización; sin embargo, para efectos de esta investigación, tanto mujeres como hombres matricularon cursos libres en ambas modalidades, esto es, por el ejemplo, los casos de baile popular, yoga, pilates para la modalidad de wellness, así como evaluaciones funcionales o entrenamiento contrarresistencia en la de fitness.

Por otra parte, en cuanto al rendimiento académico, Echavarri, Godoy y Olaz (2007) analizaron, mediante una serie de test que evalúan las aptitudes diferenciales, las diferencias que se presentan según género en cuanto a habilidades cognitivas y su relación con el rendimiento académico de estudiantes que ingresaron a la Universidad Empresarial Siglo 21. Estos test fueron aplicados a 1529 estudiantes (713 hombres y 816 mujeres), durante el periodo comprendido entre 1998 y el 2000; en los resultados se encontraron diferencias de género estadísticamente significativas, tanto en las habilidades como en el rendimiento académico.

Sin embargo, en contraposición con el párrafo inmediatamente anterior, en el análisis de datos del presente estudio no se encontraron diferencias estadísticamente significativas correspondientes a la variable aprobación en el estudiantado de los cursos libres, sino más bien se evidencia cómo el porcentaje de aprobación de hombres y el corresponiente a mujeres participantes de esta investigación son proporcionalmente similares.

Finalmente, es importante resaltar que, en el desarrollo del presente estudio, no se encontraron investigaciones similares en relación con la preferencia por sexo y la matrícula de cursos libres de PROCESA y CEDERSA para las modalidades fitness y wellness. Cabe destacar, sin embargo, que el estudio sobre las preferencias en matricula según sexo constituye un aspecto importante en cuanto a variables de oferta y demanda a futuro. Estos resultados, en su conjunto, evidencian el cumplimiento del objetivo de la universidad de mejorar las capacidades de formación de la población en cuestión. 


\section{Conclusiones}

La conclusión principal extraída de esta investigación es que, contrario a la información bibliográfica internacional, se han demostrado una serie de diferencias entre la práctica de actividad física y el sexo, en el caso de los cursos libres de los Programas Ciencias del Ejercicio y la Salud (PROCESA) y el Centro de Desarrollo y Rehabilitación de la Salud (CEDERSA) de la CIEMHCAVI, pues no se determina una diferenciación relacionada con la variable de sexo respecto a la aprobación o no de estos cursos. Asimismo, mediante los cursos libres, los esfuerzos de estos proyectos han logrado incorporar y fomentar la matrícula de ambos sexos en ambas modalidades ofertadas.

Ante ello, se puede argumentar que la CIEMHCAVI, por medio de los cursos libres que ofrecen estos programas, realiza un aporte a la construcción de una sociedad costarricense más inclusiva desde la construcción del enfoque de género, que se consolida en los principios de igualdad y equidad, al no presentar discriminación por sexo en sus cursos de educación no formal.

Tal como lo establece la Carta a la Tierra, es necesario reafirmar que cuando se posee mayor libertad de acción, conocimiento y empoderamiento, existe una mayor responsabilidad por promover el bien común y un mayor compromiso (Iniciativa Carta de la Tierra, 2000). En este sentido, la Universidad Nacional contribuye con su compromiso para que las personas que acceden a la institución se empoderen, por medio de la adquisición del conocimiento hacia una sociedad más analítica, reflexiva, crítica, equitativa, participativa y justa.

\section{Referencias}

Almorza, D., Yébenes, A., Rivas, R. y Bablé, J. A. (2010). El deporte universitario en Andalucía. Cádiz: Servicio de Publicaciones de la Universidad de Cádiz.

Anker, R. (1997). La segregación profesional entre hombres y mujeres. Repaso de las teorías. Revista Internacional del Trabajo, 116(3), 343-370.

Anker, R., Melkas, H. y Korten, A. (2003). Gender-based occupational segregation in the 1990's. Working Paper. Recuperado de http://www.ilo.org/wcmsp5/groups/public/@ed norm/@ declaration/documents/publication/wcms decl wp 18 en.pdf

Ballesteros, R. (1997). La escritora en la sociedad latinoamericana. Santiago de Cali: Universidad del Valle.

Brunet, I. y Alarcón, A. (2005). Mercado de trabajo y familia. RIPS. Revista de Investigaciones Políticas y Sociológicas, 4(2), 115-129. Recuperado de http://www.redalyc.org/html/380/38040208/ 
Cárdenas, A., Correa, N. y Prado, X. (2013). Segregación laboral y género: Tendencias y desafíos relativos al mercado laboral chileno. En A. Cárdenas (Coord.), ponencia presentada en GT, 18: Reestructuración productiva, trabajo y dominación social.

Codina, N. y Pestana, J. V. (2012). Estudio de la relación del entorno psicosocial en la práctica deportiva de la mujer. Revista de Psicología del Deporte, 21(2), 243-251. Recuperado de https://www.rpd-online.com/article/view/926/pdf

Colado, J. C. (2000). Apuntes de monitor de fitness. Madrid: FEDA.

Consejo Nacional de Rectores (CONARE). (07 de noviembre, 2013). Taller regional 2013 de estadísticas educativas de UNESCO. Sistema de Educación Superioren Costa Rica [Diapostivias en PowerPoint]. Recuperado de https://docplayer.es/18954257-Taller-regional-2013-deestadisticas-educativas-de-unesco-sistema-de-educacion-superior-en-costa-rica.html

Corrales, A. R. (2010). El fitness entendido como ocio actual saludable. TRANCES: Revista de Transmisión del Conocimiento Educativo y Transmisión del Conocimiento Educativo y de la Salud, 2(1), 14-29. Recuperado de http://www.trances.es/papers/TCS\%2002 1 2.pdf

Echavarri, M., Godoy, J. C. y Olaz, F. (2007). Diferencias de género en habilidades cognitivas y rendimiento académico en estudiantes universitarios. Universitas Psychologica, 6(2), 319329. Recuperado de http://pepsic.bvsalud.org/pdf/up/v6n2/v6n2a11.pdf

European Commission Research \& Innovation. (2011). Manual de género en la investigación. Recuperado de www.idi.mineco.gob.es/stfls/MICINN/Investigacion/FICHEROS/El genero en la investigacion.pdf

García, M. y LLopis, R. (2011). Ideal democrático y bienestar personal. Encuesta sobre los hábitos deportivos en España 2010. Madrid: CIS. Recuperado de http://www.csd.gob.es/csd/ estaticos/dep-soc/encuesta-habitos-deportivos2010.pdf

Godoy, L., Díaz, X. y Mauro, A. (2009). Imágenes sobre el trabajo femenino en Chile, 1880-2000. Revista UNIVERSUM, 24(2), 74-93. doi: https://doi.org/10.4067/S0718-23762009000200005

Iniciativa Carta de la Tierra. (2000). La Carta de la Tierra. Recuperado de https://cartadelatierra. org/descubra/la-carta-de-la-tierra/

Moreno, J. A., Martínez, C. y Alonso, N. (2006). Actitudes hacia la práctica físico-deportiva según el sexo del practicante. RICYDE. Revista Internacional de Ciencias del Deporte, 2(3), 20-43. doi: https://doi.org/10.5232/ricyde2006.00302

Naciones Unidas Secretario General. (2017). Mensaje del Secretario General con Motivo del Día Internacional de la Mujer. New York: Autor. Recuperado de https://www.un.org/sg/es/ content/sg/statement/2017-03-08/secretary-generals-written-message-internationalwomen $\%$ E2\% $80 \% 99$ s-day 
doi: http://dx.doi.org/10.15359/ree.23-3.10

URL: http://www.una.ac.cr/educare

CORREO: educare@una.cr

OECD. (2007). Understanding the social outcomes of learning. Francia: Autor. doi: https://doi. org/10.1787/9789264034181-en

Offen, K. (2000). European feminisms 1700-1950. A political history. California: Stanford University Press.

Organizción de las Naciones Unidas para la Educación la Ciencia y la Cultura (UNESCO). (2015). UNESCO Science Report: Towards 2030. France: Autor. Recuperado de https://en.unesco. org/unesco science report

Oviedo, G., Sánchez, J., Castro, R., Calvo, M., Sevilla, J. C. y Iglesias, A. y Guerra, M. (2013). Niveles de actividad física en población adolescente: Estudio de caso. Retos. Nuevas tendencias en Educación Física, Deporte y Recreación, 23, 43-47. Recuperado de https://dialnet.unirioja. es/servlet/articulo?codigo $=4135239$

Palou, P., Ponseti, X. y Borrás, P. A. (2001). Hábitos deportivos de los estudiantes de la universidad de las Islas Baleares. Educación y Cultura, 14, 163-180.

Papadópulos, J. y Radakovich, R. (2006). Educación superior y género en América Latina y el Caribe (Capítulo 8). Recuperado de https://www.cned.cl/otros-estudios/educacion-superior-ygenero-en-america-latina-y-el-caribe

PNUD. (2016). Panorama general. Informe sobre desarrollo humano 2016. Desarrollo humano para todos. Nueva York: Autor. Recuperado de http://hdr.undp.org/sites/default/files/ HDR2016 SP Overview Web.pdf

Programa Estado de la Nación. (2015). Capítulo 4: La evolución de la educación superior. En I. Román (Coord.), Quinto informe Estado de la Educación 2015 (pp. 183-247). San José, Costa Rica: Autor. Recuperado de http//:www.estadonacion.or.cr/educacion2015/assets/cap-4ee-2015.pdf

Programa Estado de la Nación. (2017). La evolución de la educación superior (Capítulo 5). En EE (Ed.), Sexto informe estado de la educación (pp. 241-308). Recuperado de http://www. estadonacion.or.cr/educacion2017/assets/parte-1-capitulo-5.pdf

Soto, M. (10 de febrero del 2017). Costa Rica se encamina hacia la paridad de género en ciencia. La Nación. Recuperado de https://www.nacion.com/ciencia/aplicacionescientificas/costa-rica-se-encamina-hacia-la-paridad-de-genero-en-ciencia/ RIPSOISWNREZXH4UAW2IDM6EDA/story/

Varela, P. (8 de Junio, 2014). Las mujeres avanzan en América Latina. El País. Recuperado de http:// www.economia.elpais.com/economia/2014/06/05/actualidad/1401989344_243833.html 